\title{
Development and Testing of a Poly-Finger Gripper for a Planetary Rover in the Fields of Science and Study
}

\author{
Tobias Planitzer (Author) \\ Department of Aeronautics and Astronautics \\ Chair of Space Technology \\ Technische Universität Berlin (TUB) \\ Berlin, Germany \\ tobias.planitzer@campus.tu-berlin
}

\author{
Lennart Kryza (Author) \\ Department of Aeronautics and Astronautics \\ Chair of Space Technology \\ Technische Universität Berlin (TUB) \\ Berlin, Germany \\ lennart.kryza@tu-berlin.de
}

\begin{abstract}
This paper describes the design of an end-effector system suited for a mars analogue mission. In the scope of a bachelors thesis, a cost-effective, lightweight and robust gripper system was developed. The system was successfully tested and verified. It will be used on a mobile robotic platform for educational and research purposes.
\end{abstract}

Keywords-rover; space robotics; gripper; cost-effective; open source; open hardware; European Rover Challenge; Mars analogue

\section{INTRODUCTION}

In the fields of space exploration, robotic systems are indispensable. Rovers utilize end-effectors to interact with their surroundings, manipulate objects or take samples. This paper elaborates an end-effector system suited for a mission scenario derived from the European Rover Challenge 2018 [1]. The BEAR Gripper offers a high grade of versatility and is inspired by anthropomorphic mechanisms of the human hand. It was designed, manufactured and tested at the Technische Universität Berlin. At the current time, it is used on the Bear Exploration and Assistant Rover (BEAR). A cost-effective, lightweight and robust gripper system was developed in the scope of a Bachelor's thesis. The ERC mission scenario served as a realistic application frame.The event focuses on robotic systems developed by students and puts them to the test, under simulated surface conditions of a foreign planetary body. In 2018, a Mars analogueenvironment was chosen as the scenario. They are best described as corresponding to assist service tasks for a manned exploration mission. These include the manipulation of a terminal by turning knobs and switches into specified positions, grasping cylindrical cache containers and obtaining various soil samples. A gripper system by OpenBionics [2] served as inspiration. Keeping the bioinspired grasping mechanism in mind, the BEAR Gripper finds a remastered way to implement an underactuated gripper system. Three fingers with two joints each are realized. They are actuated by a single servo motor [3] via a combination of steel strings and springs. Novelty lies in the implemented joint design which unites the joints and the guidance system for the steel strings. A differential mechanism prevents the fingers from any uncertainties of force application. Furthermore, it enables them to individually grasp around irregular shaped objects without dedicated commands. The gripper can be changed between a cylindrical and a spherical grasping pattern by rotating two of the fingers into an opposing orientation. The system was successfully tested and verified in all tasks. The design of the whole system is planned to be released as an open hardware design soon.

\section{DEVELOPMENT BREAKDOWN}

The BEAR Gripper was designed regarding its requirements for the numerous tasks in the given mission scenario. By analyzing the given requirements and estimating boundary conditions, five concepts have been considered. Concepts featuring cylindrical and spherical grasping patterns were compared along with configurations of two fingers up to four fingers. These concepts were evaluated using MultiAttribute-Utility [4].This method was applied on each mission scenario separately, resulting in the present three finger design with a hybrid grasping pattern. Via rapid prototyping and the principles of lightweight design commonly used in the aerospace industry [5], the design was improved iteratively. Physical properties like kinematics and dynamics were determined by calculus in mechanics and implementing into computer simulation. Stress and reliability estimations were made analytically and later tested. Most of the system was manufactured and assembled at the department's workshop. The testing took place under laboratory conditions. Therefore, each mission was simulated and success criteria had to be achieved.

\section{DETAILED DESIGN}

The anthropomorphic approach leads to the use of strings for mimicking tendons form a human hand. Thus, strings are attached at the end of each finger which are guided with pulleys through the phalanges and the rest of the gripper's casing to the actuator. The actuator is the Dynamixel MX-64 servo motor [3], which pulls the strings in or releases them. In this way, the strings are shortened and due to geometric constrains the fingers bend. This is an underactuated process 
since only one actuator operates two degrees of freedom of each finger. If one finger is blocked, a differential connecting all finger strings allows the other fingers to continue their movement, ultimately succeeding in the grasping process. This is a versatile principal to grasp numerous objects from different shapes without the need of pre-programmed grasping patterns or specialized finger shapes. It can adapt to a variety of surface geometries. To minimize and simplify the precision mechanics, the hinges of the joints simultaneously serve as guiding pulleys for the steel strings. With a pulley placed into the geometrical rotation axis of the hinge, no secondary pulleys are needed to guide the strings around the joints or align them afterwards. Additionally, the string's exposure to the open environment is reduced so no obstacle can harm (or even cut) them by direct contact over the grasping process. Fig. 1 shows the CAD of the finger with all its features.

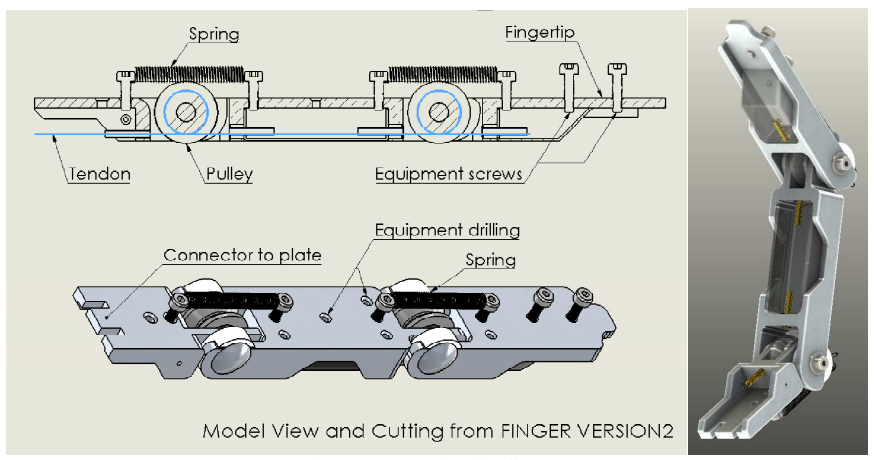

Figure 1: Robotic finger

The mechanism to open the actuated finger was implemented by springs on the outside of the finger. Contracting a finger builds up a certain spring force which pulls the phalanges back into the open starting position. The whole assembly is shown in Fig. 2. An acryl glass window allows an inside view onto the servo motor and the string guiding system.

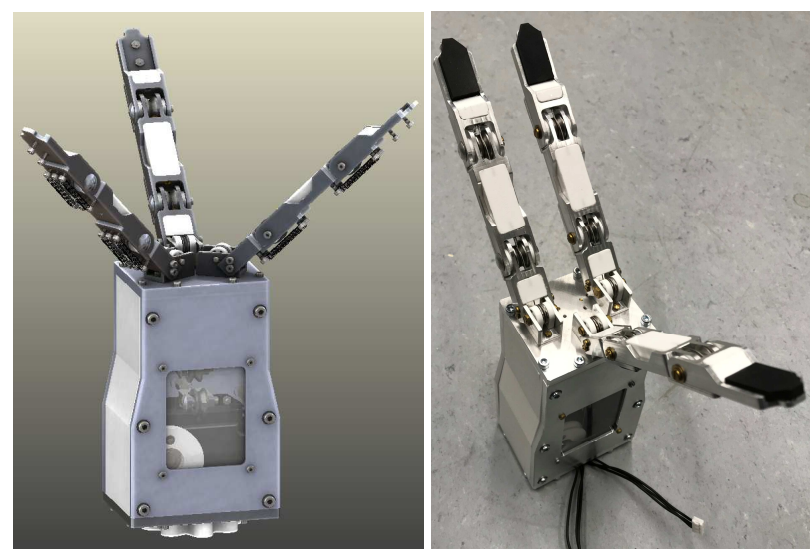

Figure 2: CAD (left) and manufactured (right) gripper system

\section{TESTING AND FUTURE STRATEGY}

Multiple experiments following the three Field Test scenarios from the ERC were conducted to prove the performance and show potential limits of the gripper. For controlling the servo motor, the software Robotis R+ Manager was utilized [7].In the scope of the Collection Task, special cache cylinders and containers should be grasped and stored. This was successfully done with replicas. The Maintenance Task posed the challenge to operate switches and knobs on a terminal interface which was ergonomically designed for humans. The tests were conducted on a terminal mockup. All interfaces could be operated. The Science Task required gathering of soil samples and subsequently storing them. For this scenario, three types of sand were used differing in their granularity. The gripper was equipped with also developed shovel attachments for the fingers. Fig. 3 displays the BEAR gripper performing and passing all three different tests without any major issues or failures.

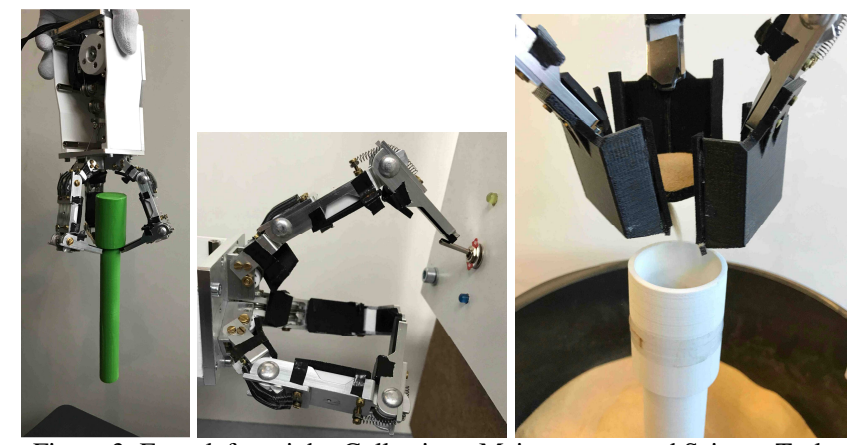

Figure 3: From left to right: Collecting-, Maintenance- and Science Task

After testing, a series of remarks arose. Getting a solid hold on small objects was difficult. Rubber strips on the fingers could increase the grip on smooth surfaces. Additionally, the differential prevented the system of building up static holding force equally for each finger. Maintaining a specific position for one stressed finger was not possible. Since the MX-64 provides a very high stall torque $(6 \mathrm{Nm})$ there still is potential for performance increase. By upgrading the steel strings' thickness or material with similar tensile strength, the maximum torque could be increased. Additionally, the counteracting springs at the fingers' outsides can be replaced by versions with a higher stiffness. This would stabilize the whole opening and closing procedure and could provide a greater static force output for the fingers. A rather large upgrade consists of the implementation of a second actuator mechanism to rotate the two fingers remotely.

\section{REFERENCES}

[1] A.M.A. Dollar, W.Bircher. "Yale Openhand Project", 2014

[2] C.I.M.A. Zisimatos, M.V. Liarokapis. "How to Create Affordable, Modular, Light-Weight, Unteractuated, Compliant Robot Hands", 2015

[3] European Rover Challenge 2018

[4] S. Jansen, "The multi-attribute utility method", p.107, 2018

[5] P. Ermanni, "Laboratory of Composite Materials and Adaptive Structures", 2015

[6] Dynamixel. "Robotis e-manual v.1.31.30.”, 2010

[7] Robotis e-Manual, "RoboPlus Manager 2.0”, 2019 